\title{
Control de la tuberculosis en un sistema penitenciario brasileño: un estudio con métodos mixtos
}

\author{
Tuberculosis control in a Brazilian prison system: a mixed methods study \\ Controle da tuberculose em um sistema prisional brasileiro: um estudo com métodos mistos
}

Mônica Cristina Ribeiro Alexandre d'Auria de $\operatorname{Lima}^{1}$ (1)

Maria Mercedes Martinez-Marcos² (1) Jaqueline Garcia de Almeida Ballestero ${ }^{3}$ (1) Teresinha Heck Weiller ${ }^{4}$ (1)

Cassiara Boeno Borges de Oliveira $^{3}$ (1) Pedro Fredemir Palha ${ }^{3}$ (1)

\begin{abstract}
1. Universidade de São Paulo, Escola de Enfermagem de Ribeirão Preto, Programa de Pós-graduação Enfermagem em Saúde Pública. Ribeirão Preto, SP, Brasil.
\end{abstract}

2. Universidad Autónoma de Madrid, Facultad de Medicina, Seccion Departamental de Enfermería. Madri, Espanha.

3. Universidade de São Paulo, Escola de Enfermagem de Ribeirão Preto. Ribeirão Preto, SP, Brasil.

4. Universidade Federal de Santa Maria, Departamento de Enfermagem. Santa Maria, RS, Brasil.

Autor correspondiente: Mônica Cristina Ribeiro Alexandre d'Auria de Lima.

E-mail: mcraadl@gmail.com.

Recibido en 09/03/2021.

Aprobado en 11/08/2021.

DOl:https://doi.org/10.1590/2177-9465-EAN-2021-0068

\begin{abstract}
Resumen
Objetivo: analizar el control de la tuberculosis en un sistema penitenciario de un Estado brasileño. Método: estudio mixto explicativo secuencial. Fase I: descriptiva retrospectiva de los casos de tuberculosis en presos del estado de São Paulo en el periodo 2010-2016. Los casos se analizaron con frecuencias simples en el software SPSS 20.0 de la IBM y tendencia temporal en el software Stata/SE 14.0, por auto regresión de Prais-Winsten. Fase Il: cualitativa. Se realizó con seis informantes-clave (profesionales sanitarios de una prisión) con base en la Teoría Fundamentada Constructivista. Resultados: de 16.640 casos, 95,8\% eran pulmonares, la Demanda Ambulatoria posibilitó el diagnóstico del 51,4\% y la curación fue la conclusión de tratamiento más frecuente. La categoría central fue: "Hacer bien su trabajo" $y$ las subcategorías fueron: "Descubrir la enfermedad" e "Implicarse en el tratamiento". Éstas señalaron las estrategias y acciones desarrolladas por los profesionales para diagnosticar y tratar a los presos enfermos. Conclusión e implicaciones para la práctica: los resultados sugieren una importante situación de la tuberculosis en penitenciarías, lo que trae consigo la necesidad de mejor articulación con el equipo de seguridad para un adecuado desarrollo de las estrategias que posibilitan un diagnóstico temprano y un tratamiento adecuado.
\end{abstract}

Palabras claves: Tuberculosis; Prisiones; Prisioneros; Diagnostico; Terapia por Observación Directa.

\begin{abstract}
Objective: to analyze tuberculosis control in a prison system of a Brazilian state. Method: this was a sequential explanatory mixed study. In phase I, a quantitative retrospective description was conducted of recorded tuberculosis cases among prisoners in the state of São Paulo from 2010-2016, which were analyzed using simple frequencies with IBM SPSS 20.0 software and time-trend with Stata software/SE 14.0 via Prais-Winsten regression. Phase II was qualitative and was based on interviews with six key informants (health professionals working at a prison). Data analysis took place according to the procedures set forth in constructivist grounded theory. Results: of 16,640 cases of tuberculosis, $95.8 \%$ were pulmonary, outpatient care accounted for the diagnosis in $51.4 \%$ of cases, and tuberculosis cure was the most frequent treatment outcome. "Doing your job well" emerged as the central category, while the subcategories: "discovering the disease" and "getting involved in treatment" described the strategies and actions developed by the professionals to diagnose and treat sick prisoners. Conclusions and implications for practice: the results indicate the significant presence of tuberculosis in penitentiaries and the need to better coordinate the actions of security teams with health staff to develop adequate strategies that allow for early diagnosis and proper treatment.
\end{abstract}

Keywords: Tuberculosis; Prisons; Prisoners; Diagnosis; Directly Observed Therapy.

\section{Resumo}

Objetivo: analisar o controle da tuberculose em um sistema prisional de um Estado brasileiro. Método: estudo sequencial, misto, explanatório: fase I - retrospectiva descritiva em que foram incluídos todos os casos de tuberculose em presidiários de um Estado brasileiro, período 2010-2016, e analisados por frequências simples, no software IBM SPSS 20.0, e tendência temporal, no Software Stata / SE 14.0, por autorregressão de Prais-Winsten; Fase II - qualitativa, realizada com seis informantes-chave (profissionais de saúde de uma prisão), e a análise ocorreu segundo os procedimentos da Teoria Fundamentada Construtivista. Resultados: dos 16.640 casos, 95,8\% eram pulmonares, a Demanda Ambulatorial permitiu o diagnóstico de 51,4\%, e a cura foi a finalização de tratamento mais frequente. "Fazer bem o seu Trabalho" foi apresentado como categoria central, e as subcategorias, "Descobrindo a doença" "Envolvendo-se no tratamento", indicaram as estratégias e ações desenvolvidas pelos profissionais para diagnosticar e tratar os presos enfermos. Conclusão e implicações para a prática: os resultados sugerem uma situação importante da tuberculose nas prisões, sendo necessária uma melhor articulação com a equipe de Segurança para o desenvolvimento adequado de estratégias que permitam um diagnóstico precoce e tratamento adequado.

Palavras-chave: Tuberculose; Prisões; Prisioneiros; Diagnóstico; Terapia Diretamente Observada. 


\section{INTRODUCCIÓN}

La tuberculosis (TB) es una enfermedad infectocontagiosa que afectó a aproximadamente 10 millones de personas alrededor del mundo en el año 2018. Dichas estadísticas se han mantenido en estos últimos años ${ }^{1}$. En las penitenciarías, la TB se relaciona por hecho de convivir con un elevado número de individuos pertenecientes a grupos que presentan mayor vulnerabilidad para desarrollar la enfermedad, así como por tratarse de instituciones cerradas con deficiencias higiénicosanitarias. Adicional a esto, las condiciones de hacinamiento facilitan la transmisión de la infección ${ }^{2,3}$.

A nivel mundial, 9,9 millones de personas están en situación de privación de la libertad, gran parte de ellas en prisiones ${ }^{1}$. La población carcelaria brasileña ocupa la cuarta posición en el mundo y se sabe que de todos los casos de TB en el país, ésta se manifiesta aproximadamente en el $11 \%$ de los reclusos ${ }^{4,5}$, lo que supone un problema relevante. Adicional a lo anterior, se tiene conocimiento de que los presos mantienen contacto con la sociedad fuera del presidio, bien sea por las visitas de sus seres queridos o en el momento en que recobran la libertad.

Por lo anterior, desde el año 2003, el Ministerio de Salud (MS) de Brasil está implantando un programa específico para el control de la TB en prisiones brasileñas. La implementación de este programa de control de TB ha sido difícil, como lo pone en evidencia un estudio realizado. Dicho estudio permite observar que es notable la dificultad de acceso de los presos a los servicios de salud de la Institución y a los de la sociedad en general, debido a la escasa inversión de recursos financieros, así como la necesidad de coordinación entre los ministerios de justicia y de salud ${ }^{6}$.

Tras una Revisión de la literatura, en un estudio realizado en Portugal, se identificó que la mayor dificultad para controlar la TB en sus prisiones es el hacinamiento, seguido de la falta de recursos humanos, materiales y económicos, así como las dificultades para vincular los equipos de salud y seguridad ${ }^{7}$.

Sin embargo, lo que se relaciona más directamente con el escenario brasileño es que no hay investigaciones que abarquen en sus propósitos de investigación comprender el desarrollo del manejo del control de la TB en prisiones del país y los problemas que enfrentan los profesionales de salud para llevar a cabo el Programa de Control de TB.

A lo largo de los años, con respecto al problema de la TB en las prisiones brasileñas, se han realizado diferentes investigaciones sobre las características epidemiológicas, clínicas y sociodemográficas de la TB en los reclusos, los factores de riesgo para el desarrollo de la enfermedad, así como factores relacionados con la finalización del tratamiento o sobre la susceptibilidad y los genotipos de la TB en la población penitenciaria ${ }^{6,8,9}$.

En relación con lo que se menciona, son inexistentes investigaciones direccionadas específicamente a la obtención de una mayor comprensión de la magnitud del problema de la TB en prisiones brasileñas, cómo también el desarrollo de las acciones para su control en dichas instituciones. Lo anterior ayudaría a identificar mucho mejor los puntos frágiles y destacar puntos potenciales para la realización de dichas acciones.

Por ese motivo, el presente estudio fue desarrollado con el fin de tener una comprensión más amplia y más profunda de los acontecimientos del control de la TB en las prisiones ${ }^{10}$. El estudio busca, igualmente, proponer nuevas estrategias que posibiliten mejorar el control de esta enfermedad en las prisiones que conduzcan a posibles cambios en las directrices propuestas por el MS en su Programa de Control de la TB, y busca que correspondan con la realidad del desarrollo de las acciones de control existentes en las prisiones brasileñas.

Puesto que se trata de un estudio innovador e importante, se formuló la siguiente pregunta problema:¿cómo está el desarrollo del control de la tuberculosis en un sistema penitenciario brasileño?, con el objetivo de analizar el control de la tuberculosis en un sistema penitenciario de un Estado brasileño.

\section{MÉTODO}

Para alcanzar el objetivo propuesto, se desarrolló un estudio mixto explicativo secuencial o estudio de abordaje de seguimiento cualitativo. El estudio se dividió en dos fases (Fase I: cuantitativa y Fase II: cualitativa), con prioridad en la fase cualitativa. Se trata un estudio de metodología, denominado "quan $\rightarrow$ QUAL". ${ }^{10,11}$

Esta investigación se desarrolló en el Sistema Penitenciario del estado de São Paulo, que es el Estado brasileño con mayor número de presos del país, y representa un $36 \%$ del total de presos en el territorio nacional. En el año 2017, cuando fueron recolectados los datos para esta investigación, ya existían 170 prisiones y se preveían más de 13 prisiones a ser inauguradas en el estado de São Paulo 4 .

Para la fase I, cuantitativa, se realizó un estudio descriptivo retrospectivo del control de la TB proveniente de los datos secundarios de los casos de TB de las prisiones del estado de São Paulo. En la fase II se llevó a cabo un estudio cualitativo a través de entrevistas con profesionales sanitarios que hacían referencia al control de la TB desarrollado en una prisión en este mismo Estado.

En la fase I se incluyeron todos los casos de presos notificados con TB de las prisiones del estado de São Paulo en el periodo de 2010-2016. Los datos de la fase I se obtuvieron a través del Centro de Vigilancia Epidemiológica del Estado en enero de 2017, a través de una plantilla electrónica del Microsoft Excel $^{\circledR} 2013$. Las variables de la fase I del estudio fueron: año de notificación, tipo de caso (nuevo, retratamiento, recidiva), forma clínica (pulmonar; extrapulmonar + diseminada), condiciones de salud ( $\mathrm{VIH} /$ sida, alcoholismo drogadicción),forma que posibilitó el diagnóstico (demanda ambulatoria, búsqueda activa, urgencia/ emergencia, elucidación diagnóstica en internación, búsqueda activa de contactos, descubierta posterior al óbito), tipo de tratamiento (autoadministrado, tratamiento directamente observado), finalización del tratamiento (curación, abandono, resistencia, óbito $\mathrm{TB}$, óbito no $-\mathrm{TB}$ ).

Las variables fueron analizadas y los resultados se presentan tabulados (Tabla 1 y 2 ) en la sección "Resultados". 
Para eso, se llevó a cabo un análisis descriptivo de las variables, con frecuencias absolutas y relativas; media y desviación estándar a través software Statistical Package for the Social Sciences (SPSS) 20.0

Se realizó el cálculo de tendencia temporal para las variables: "condiciones de salud" y "finalización del tratamiento". Para ello, se calculó, en primer lugar, las incidencias anuales de "condiciones de salud", utilizando como denominador la población reclusa del Estado, cada año del período estudiado. La anterior información fue concedida por el Comité de Ética de la Secretaría de Administración de Penitenciarias del Estado (CEPSAP). Igualmente, se calculó las incidencias de la "finalización del tratamiento", utilizando como denominador el total de casos nuevos de la enfermedad anualmente (periodo de estudio) en presos del Estado.

De esta manera, para clasificar la tendencia temporal, las incidencias de dichas variables fueron logaritmitizadas con base 10 y analizadas en el software Stata/SE 14.0, también, fue utilizado el método de autoregresión de Prais-Winsten, el cual corrige la autocorrelación temporal de primer orden en el análisis de series de valores organizados en el tiempo ${ }^{12}$, y considera un intervalo de confianza de $95 \%$. Las tendencias para cada grupo supracitado están clasificadas en estacionarias, de ascendencia o de decrecimiento.

La fase II del estudio se llevó a cabo en una única prisión del estado de São Paulo, la cual se seleccionó por presentar el mayor número de reclusos, y cuenta con un régimen carcelario cerrado y semiabierto. El equipo sanitario estaba conformado por 12 profesionales: el director de la institución, el psicólogo, dos enfermeros, el técnico de enfermería, cuatro auxiliares de enfermería, el agente carcelario de sanidad, el médico clínico y dentista. El nombre de la prisión investigada en la fase II será mantenido en anonimato, para evitar posible identificación de los profesionales.

La selección de los participantes de la fase II se realizó por muestreo con propósito ${ }^{13}$. Se escogió a los profesionales que ejecutaban las acciones para el manejo de la TB en la prisión elegida para el estudio y se tuvo en cuenta como criterio de selección que el profesional se haya desempeñado en su labor al menos por un periodo de seis meses. De esta forma, se conformó un grupo con un total de 6 profesionales sanitarios.

Con los preceptos metodológicos de una investigación de método mixto explicativo secuencial ${ }^{11}$, a partir del análisis de los datos de la fase I, se identificaron importantes resultados que facilitaron el desarrollo de la fase II. En la segunda fase se realizó una encuesta semiestructurada relacionada con el abordaje del diagnóstico y tratamiento de la TB en esta Institución, a partir de las siguientes preguntas orientadoras: ¿Cuáles son las acciones de diagnóstico y tratamiento de la TB que se desarrollan en esta institución?; ¿Cómo se realizan las acciones de diagnóstico y tratamiento de la TB?; ¿Cómo es el manejo del caso de TB diagnosticado?; ¿Cómo es su experiencia con el tratamiento de la TB en la institución? Estas mismas preguntas orientadoras permitieron la formulación de otra, por parte del investigador durante las entrevistas, con base en las respuestas de los participantes.

Los participantes fueron contactados directamente en la prisión en el mes de abril del 2017. El día y hora fueron previamente acordados con los mismos y con la aprobación del director de la Institución. Las entrevistas fueron realizadas por la investigadora principal del estudio, sin presentar vínculo con los entrevistados, en un despacho privado en la sección de enfermería.

Las entrevistas realizadas fueron audio-grabadas, con duración de 60 a 100 minutos, y por lo mismo, las respuestas de los participantes fueron transcritas en su totalidad por la investigadora principal, y comprobadas con las grabaciones para verificar su exactitud. El análisis de las entrevistas transcritas se hizo de manera cualitativa, siguiendo los procedimientos de la Teoría Fundamentada Constructivista (TFC) ${ }^{14}$, y se llevó a cabo manualmente, sin utilizar un software. Cabe destacar que, además de estos datos adquiridos en las entrevistas, se suman a los mismos datos en la sombra ${ }^{15}$, adquiridos con profesionales sanitarios que trabajan en otra prisión del Estado.

De acuerdo con los procedimientos de la TFC para la codificación abierta, se analizaron los datos línea a línea, se identificaron códigos relacionados al proceso de control de la TB que realizaban los profesionales tales como: "realizar pruebas" y "aprovechar oportunidades". En la codificación focalizada se agruparon los códigos identificados en el microanálisis, tales como "indagar sobre los síntomas" y "garantizar el tratamiento", y posteriormente, se desarrollaron las categorías. El proceso de análisis y validación de las categorías de investigación fue realizado por la investigadora principal (primer autor) y el segundo autor de este estudio, quienes discutieron y validaron las categorías. El muestreo teórico se llevó a cabo para refinar y elaborar las categorías emergentes.

El método de comparación constante se utilizó en cada momento del análisis, los datos fueron comparados en las entrevistas y entre las entrevistas, lo que permitió identificar similitudes y diferencias ${ }^{16}$. Durante el análisis se elaboraron memos analíticos ${ }^{16}$ y se consultó bibliografía para apoyar la construcción de las categorías. Esta investigación se basa en los criterios de credibilidad, originalidad, resonancia y utilidad, de forma acorde con la TFC ${ }^{14}$.

Para preservar el anonimato de los participantes de las entrevistas, a cada uno de ellos se le asignó una letra "E" (Entrevistado) y un número comprendido entre 1 y 6 , los cuales corresponden al orden en que las entrevistas fueron realizadas.

El estudio fue aprobado por los Comités Éticos de la Institución de Educación Superior, con número de aprobación CAAE: 58412716.0.0000.5393 y de la SAP, número CAAE: 58412716.0.3001.5563. Se hizo la solicitud de no aplicar el Consentimiento Informado $(\mathrm{Cl})$ para la fase I, y todos los participantes del estudio de la fase II lo hicieron de manera voluntaria y se obtuvo el Cl firmado. Se garantizó los aspectos éticos y legales de investigación con seres humanos establecidos por la Resolución no 466/2012 del "Conselho Nacional de Saúde e Complementares". 


\section{RESULTADOS}

En un período de siete años (2010-2016), fueron notificados un total de 16.640 casos de prisioneros con TB en el Estado, con un cambio de incidencia de 869,9 casos $/ 100.000$ habitantes para el (2010) y 1015,2 casos/100.000 habitantes para el (2016), donde el $95,8 \%$ eran casos pulmonares.

La mayoría de los enfermos eran hombres $(97,9 \%)$, pertenecientes a población autodeclarada negra $(47,5 \%$ ) y edad media de 30 años (mediana 29 años y desviación estándar de 7,9 años). Del total de los casos diagnosticados, el 79\% eran casos nuevos y para más del $50 \%$ de los enfermos se obtuvo el diagnóstico a través de la Demanda Ambulatoria, seguido de la Búsqueda Activa de casos sintomáticos respiratorios, conforme la Tabla 1.

Con respecto a las condiciones de salud de los enfermos, el uso de drogas lícitas e ilícitas presentaron una tendencia creciente entre los casos diagnosticados en el período de estudio, luego, la coinfección por el $\mathrm{VIH} /$ sida estuvo presente en casi el $10 \%$ de los casos que todavía presentó tendencia decreciente en el respectivo período.

En el total de casos el fin del tratamiento de la enfermedad fue predominante con un $88 \%$ de curación, que, en el respectivo período, presentó una tendencia creciente, seguida del abandono de tratamiento $(6,7 \%)$.

En la Tabla 2 se muestra el porcentaje de casos y las tendencias temporales referentes a las condiciones de salud y finalización del tratamiento.

Con relación al Tratamiento Directamente Observado (TDO), fue realizado por un $90 \%$ de los casos; el 3,7\% fue Autoadministrado, y para el $6,3 \%$ se desconoce el tipo de tratamiento recibido.

Referente al estudio cualitativo, los profesionales sanitarios participantes fueron todos personal del área de enfermería, como los tres auxiliares de enfermería, un técnico de enfermería, dos enfermeros, en su mayoría mujeres (66,6\%), cuya edad estaba comprendida entre $27-68$ años y quienes contaban con tiempo de experiencia de 5 a 20 años en el sistema penitenciario.

Tabla 1. Distribución del perfil de las características clínicas, según tipo de caso y forma que posibilitó el diagnóstico de los casos de tuberculosis en presos del sistema penitenciario del estado de São Paulo del período 2010 al 2016, São Paulo-SP, Brasil.

\begin{tabular}{|c|c|c|c|}
\hline \multicolumn{2}{|c|}{ Características Clínicas y de Descubierta de la enfermedad $(n=16.640)$} & Período 2010 - 2016 (n) & $\%$ \\
\hline \multirow{3}{*}{ Tipo de caso } & Nuevo & 13.151 & 79,0 \\
\hline & Recidiva & 2.316 & 13,9 \\
\hline & Retratamiento ${ }^{a}$ & 1.173 & 7,0 \\
\hline \multirow{7}{*}{ Forma que posibilitó el diagnóstico } & Demanda Ambulatoria & 8.562 & 51,4 \\
\hline & Búsqueda Activa & 5.188 & 31,1 \\
\hline & Urgencia / Emergencia & 922 & 5,5 \\
\hline & Elucidación Diagnóstica en Internación ${ }^{b}$ & 789 & 4,7 \\
\hline & Búsqueda Activa de Contactos & 784 & 4,7 \\
\hline & Sin información & 360 & 2,1 \\
\hline & Descubierta posterior al óbito & 35 & 0,2 \\
\hline
\end{tabular}

aCasos de retratamiento por abandono, resistencia medicamentosa o cambio de tratamiento. ${ }^{\mathrm{b} C a s o s}$ en Internación en Hospital Penitenciario y/o en Hospital Público.

Fuente: Centro de Vigilancia Epidemiológica de la Secretaría de Salud del Estado. Sistema de información de tuberculosis.

Tabla 2. Distribución y tendencia temporal del perfil de los casos según condiciones de salud y finalización de tratamiento de los casos de tuberculosis en presos del sistema penitenciario de un estado de São Paulo, período 2010 al 2016 , São Paulo-SP, Brasil.

\begin{tabular}{|c|c|c|c|c|}
\hline Perfil de los casos & $\begin{array}{c}\text { Período } 2010-2016 \\
\text { (n) }\end{array}$ & $\%$ & $\begin{array}{l}\text { Período } 2010 \text {-2016 } \\
\text { Coeficiente [IC 95\%] }\end{array}$ & Tendencia Temporal \\
\hline \multicolumn{5}{|c|}{ Condiciones de Salud } \\
\hline VIH/sida & 1.423 & 8,6 & $-0,046[-0,062 ;-0,030]$ & Decreciente \\
\hline Alcoholismo & 1.032 & 6,2 & $0,077[0,055 ; 0,098]$ & Creciente \\
\hline Uso drogas ilícitas & 2.753 & 16,5 & $0,055[0,037 ; 0,074]$ & Creciente \\
\hline \multicolumn{5}{|c|}{ Finalización del tratamiento } \\
\hline Curación & 14.637 & 88,0 & $0,001[0,000 ; 0,002]$ & Creciente \\
\hline Abandono & 1.122 & 6,7 & $-0,026[-0,041 ;-0,011]$ & Decreciente \\
\hline Resistencia & 199 & 1,2 & $0,005[-0,046 ; 0,058]$ & Estacionaria \\
\hline Óbito TB & 78 & 0,5 & $0,021[-0,012 ; 0,056]$ & Estacionaria \\
\hline Óbito no -TB & 252 & 1,5 & $-0,099[-0,116 ;-0,081]$ & Decreciente \\
\hline
\end{tabular}

Fuente: Centro de Vigilancia Epidemiológica de la Secretaría de Salud del Estado. Sistema de información de tuberculosis. 
"Hacer bien su trabajo" es la categoría central que describe el control de la TB por los profesionales para llevar a cabo un adecuado manejo de la enfermedad y atención a los presos enfermos, como parte esencial de su labor profesional. Las dos subcategorías: "descubrir la enfermedad" e "implicarse en el tratamiento de la enfermedad", forman parte del proceso que involucra hacer bien su trabajo, en un contexto penitenciario que impone límites para desarrollar dicho trabajo, bajo las condiciones en las que se encuentran los presos, condiciones que no favorecen la detección y tratamiento de la enfermedad. En las subcategorías se presentan las estrategias implementadas por los profesionales, en las cuales desarrollan las acciones para el control de la TB en el proceso de atención a los enfermos (Tabla 3).

Descubrir la enfermedad es el primer paso para el control de la TB; de hecho, hay dos estrategias que lo posibilitan: "indagar en lo oculto" e "indagar en lo manifiesto". El diagnóstico de una enfermedad como la TB no siempre es algo sencillo, es más difícil en un entorno penitenciario, y se convierte en un ejercicio de hallazgo, que puede estar oculto, o ser desconocido para los reclusos que padecen de la enfermedad.

Indagar en lo oculto es una estrategia que posibilita a los profesionales sanitarios descubrir el estado de salud que presentan los presos y aprovecha la presencia de los presos en la enfermería para valorar los síntomas de la enfermedad. Los profesionales son conscientes que algunos presos pueden estar enfermos de TB pero no solicitan atención, y si acceden a la enfermería, no verbalizan sus síntomas; por eso es importante para ellos aprovechar cualquier contacto que mantienen con los presos en la enfermería, a fin de indagar sobre los signos y síntomas más característicos de la TB, principalmente la tos.

También son conscientes de que no pueden tener contacto con los presos en sus celdas por los límites que impone el equipo de seguridad de la prisión, y esto dificulta las posibilidades de detectar la enfermedad. Así que, para ellos, es muy importante que las personas que forman parte del entorno penitenciario estén concienciadas e informadas sobre los síntomas de la TB y de la importancia del diagnóstico. Para ello cuentan con la ayuda de los agentes de seguridad y de los presos en celda, como señaló este participante:
[...] Entonces si él [preso] ve que el compañero de celda no está bien, tiene todos los síntomas, [...] avisa al guardia, y ellos traen al enfermo para acá (E2).

Otra acción que llevan a cabo los profesionales para identificar los casos de TB, es con la colaboración de los agentes de seguridad, quienes llevan a los presos en sus celdas por dos veces al año los cuestionarios elaborados por los profesionales sanitarios, para que los presos señalen si presentan los síntomas de la enfermedad tales como la tos, fiebre nocturna y/o adelgazamiento:

[...] Hace la búsqueda activa en la celda y tomamos allí los síntomas, si están con fiebre, adelgazando, está con tos $[\ldots]$ (E5).

Los participantes expresaron que descubrir el preso que está enfermo exige de su empeño y búsqueda constante, así que, cuando se descubre y se diagnostica a un caso positivo de TB esto implica la búsqueda activa de sus contactos. Lo anterior se hace con el objetivo de garantizar que no quede sin diagnosticar ningún preso enfermo, así que, a todos los presos que han convivido en la celda con el preso enfermo se les realiza el examen diagnóstico. Sin embargo, los profesionales saben que solamente es posible indagar a los contactos que compartían celda en el momento del diagnóstico, ya que, por motivos de seguridad, los presos cambian de celdas constantemente y esto dificulta su trabajo.

"Indagar en lo manifiesto" es la estrategia en la que los profesionales van a percibir las manifestaciones clínicas de la TB entre los presos que acceden a la enfermería, discriminando cuáles son los presos cuyos síntomas efectivamente corresponden con la enfermedad de la TB de aquellos otros presos que presentan síntomas coincidentes a otras condiciones de salud. Los profesionales son conscientes de las condiciones de salud que comúnmente presentan los presos, tales como la presencia del $\mathrm{VIH} /$ sida o la drogadicción, que pueden favorecer el adelgazamiento, fiebre, y también favorecen la presencia de la tos. Esto requiere que, ante todos los casos de sospecha, se soliciten pruebas para confirmar el diagnóstico:

Tabla 3. Subcategorías y estrategias implementadas por los profesionales sanitarios en el proceso de atención a los enfermos por tuberculosis, bajo la Categoría Central, 2021, São Paulo-SP, Brasil.

Categoría Central: Hacer Bien su Trabajo

\section{Subcategorías}

Descubrir la enfermedad

\begin{tabular}{cc} 
Subcategorías & Estrategias \\
\hline Descubrir la enfermedad & $\begin{array}{c}\text { Indagar en lo oculto } \\
\text { Indagar en lo manifiesto }\end{array}$ \\
Implicarse en el tratamiento de la enfermedad & Persuadir para que se inicie el tratamiento \\
& Orientar sobre el cuidado y tratamiento \\
\hline
\end{tabular}

Fuente: Autoría propia. 
[...] Digamos que él está presentando solamente la tos, o solamente fiebre, o sea, presentaba un único síntoma y ahí resolvimos hacer la solicitud de una baciloscopia, por peso de consciencia [...] (E1).

Los profesionales son conscientes de que no pueden y no deben minusvalorar los signos y síntomas que los presos relatan cuando están presentes en la enfermería, todo esto por motivos de seguridad y hacinamiento, lo que dificulta el acceso a la enfermería por parte de los presos. Es por ello, que para los profesionales, esta es una oportunidad que tienen para poder realizar un diagnóstico, de modo que dedican el tiempo necesario para atenderlos y solicitar las pruebas necesarias.

Además del diagnóstico, los participantes del estudio se implican en el tratamiento de la enfermedad, colocando las estrategias que desarrollan en función de las diferentes etapas que requiere del tratamiento de la TB y del compromiso que los presos expresan para cumplir el tratamiento (Figura 1).

Al inicio del tratamiento, se requiere aislamiento del preso bacilífero en la enfermería y su seguimiento se hace de manera ambulatoria. Por motivos de dificultades por parte del equipo de seguridad en garantizar el traslado todos los días para recibir la medicación de la TB del preso en la enfermería, se recurre al aislamiento respiratorio en la enfermería por al menos 15 días, lo que se constituye en esta prisión cómo el único momento que los profesionales realizan el TDO para los enfermos.

Sin embargo, a los reclusos no les gusta el aislamiento en la enfermería, ya que supone la interrupción de su convivencia con los otros presos, así como de su derecho a visitas y actividades de ocio o tiempo libre. Los profesionales sanitarios identifican a los presos que son complacientes con el tratamiento de su enfermedad y utilizan la estrategia de persuadir para iniciar su tratamiento. Persuaden a los presos para que acepten el aislamiento necesario para lo cual los profesionales sanitarios argumentan a los reclusos que el aislamiento no es una situación muy negativa, ya que se pueden beneficiar de la atención y disposición brindada por parte de los profesionales de la enfermería cuando lo deseen:

[...] Conversamos con él, les decimos que vamos a estar a su disposición [...] y que él va a tenernos muy cerca, quince días o más [...] (E4).

Para un adecuado control de la TB, el aislamiento es necesario y parte inicial del tratamiento, no existe la posibilidad de que el preso elija si quiere o no ser aislado. Cuando los presos enfermos no demuestran interés en cumplir o no quieran iniciar su tratamiento, los profesionales sanitarios deben obligarlos de manera coercitiva a que inicien el aislamiento en la enfermería y

INICIO DEL TRATAMIENTO

AISLAMIENTO

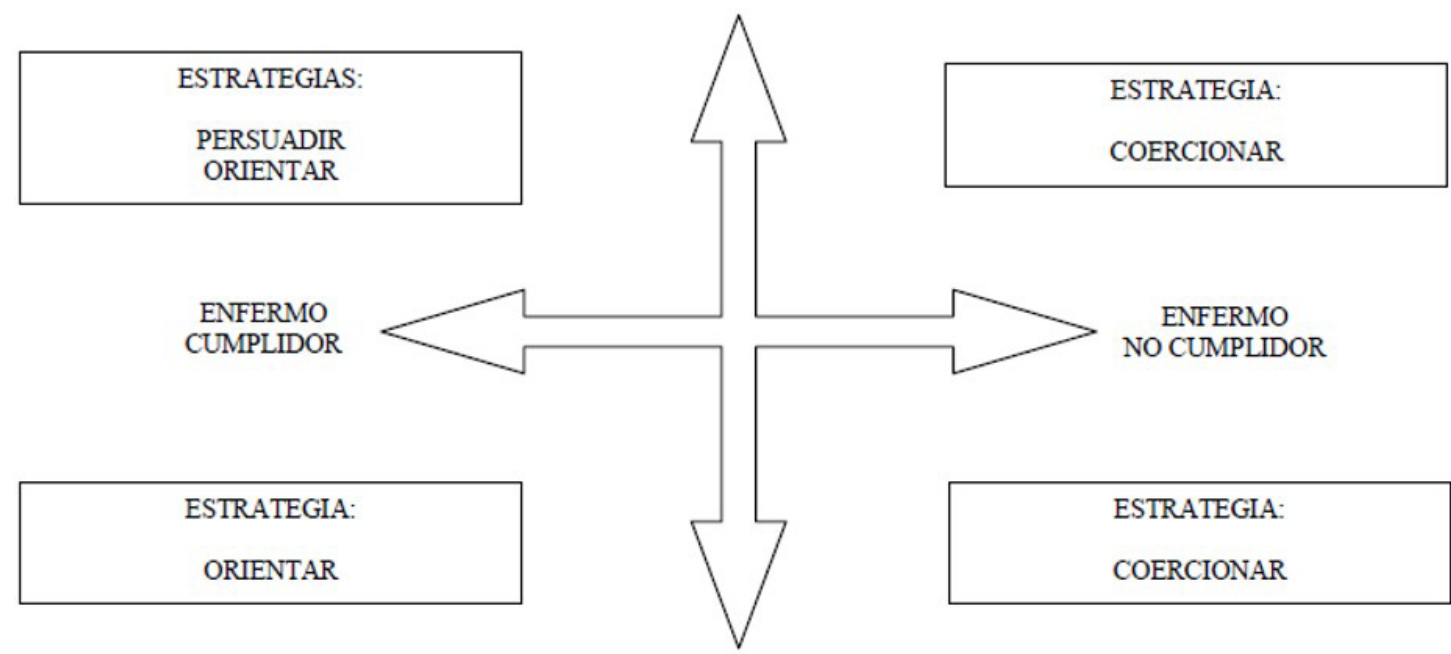

MANTENER TRATAMIENTO

CONSOLIDAR TRATAMIENTO

Figura 1. Estrategias desarrolladas por los profesionales sanitarios de una prisión del estado de São Paulo, conforme al momento del tratamiento de la enfermedad y compromiso del preso. São Paulo, 2021.

Fuente: Autoría propia. 
cumplan el tratamiento de manera ambulatoria. De modo que, para estos presos, se utiliza la estrategia de coercionar para cumplir el tratamiento

La coerción, significa que los profesionales ejercen una presión sobre los presos para forzar su voluntad y su conducta; utilizan la coerción haciendo énfasis y presión en las consecuencias que tendrán para ellos no iniciar o llevar a cabo su tratamiento como: restringir sus actividades de ocio o tiempo libre y/o perder su derecho a visitas. Por lo tanto, se deben hacer pruebas de baciloscopia durante el inicio del tratamiento para no perder estos beneficios, como señaló este participante:

[...] Solamente saldrá del aislamiento cuando se empiece a tratary el examen de baciloscopia salga negativo [...] (E1).

Los profesionales de la salud expresan con contundencia las consecuencias que tendrá para su salud y citan expresamente la muerte por la enfermedad, como lo ha expresado este participante:

"Les avisamos que es una enfermedad letal si no es tratada correctamente.” (E6).

En esta situación de aislamiento, los profesionales mantienen al menos 15 días de contacto con los presos, con mucha proximidad y ofrecen el TDO. El aislamiento brinda a los profesionales la oportunidad de desarrollar la estrategia de orientar sobre el cuidado y tratamiento, educando a los presos sobre la toma adecuada del medicamento, qué deben realizar durante su tratamiento ambulatorio cuando regresen a convivir en las celdas, sin el TDO.

En el proceso del tratamiento de manera ambulatoria, los profesionales comprenden que es necesario implicar y responsabilizar al preso enfermo sobre el cumplimiento del tratamiento, para ello, siguen con la estrategia de orientar sobre el cuidado y la importancia de tratarse. Además, saben que los presos mantienen hábitos que pueden perjudicar el tratamiento y su salud, por lo que es necesario orientarlos a que sigan hábitos más saludables, como no utilizar o disminuir el uso de drogas con el fin de lograr una curación mucho más fácil.

Sin embargo, el cumplimiento del tratamiento que los presos llevan de manera ambulatoria, no siempre resulta fáci de conseguir. Cuando los profesionales, conscientes de que no pueden obligar a los presos a cumplir con el tratamiento, notan que un enfermo no está llevando a cabo el tratamiento y la orientación sobre los cuidados no resulta efectiva, recurren nuevamente la estrategia de coerción.

Para los profesionales lo más importante es que los presos enfermos se curen, como también interrumpir la cadena de trasmisión de la enfermedad y controlar la enfermedad. Los profesionales no serán complacientes con aquellos presos que no quieren tratamiento, lo que los lleva a recurrir nuevamente a la coerción con la amenaza de volver al aislamiento y permanecer así hasta que se realice el tratamiento:
[...] Hace parte del tratamiento tener la cura total [tomar la medicina]. Pero ahora, cuando hay esos casos [que no se quieren tratar], los traemos aquí en la enfermería y mientras él no se empiece a tratar correctamente, no los dejamos libres para que vuelva a convivir con los otros presos [...](E6).

Los profesionales sanitarios afirman que coercionar es la estrategia más agresiva, y la llevan a cabo para no dejar que los presos perjudiquen el proceso de atención para el control de la enfermedad, y así cumplir con su misión en hacer bien su trabajo.

\section{DISCUSIÓN}

La magnitud de la TB en el sistema carcelario del Estado estudiado es importante; la incidencia de TB es mayor de 850 casos/100.000 presos, llegando a más de 1.000 casos/100.000 presos. Este aumento puede ser debido al gran número de casos pulmonares, que, posiblemente, no se diagnostican de manera rápida y/o no se tratan correctamente ${ }^{2}$ La presencia de casos de recidiva y retratamiento supone la dificultad de tratar correctamente a los presos enfermos en las prisiones, lo que refleja una perpetuación de exposición al bacilo de la TB. Se trata de un escenario que puede favorecer a la propagación de la TB multirresistente, agravando el problema ${ }^{17}$.

Los resultados cualitativos, ayudan para una comprensión sobre el panorama de los casos identificados en el sistema penitenciario del Estado. No obstante, este estudio muestra que aunque la prisión estudiada está compuesta por un menor número de profesionales sanitarios que lo recomendado por el $\mathrm{MS}^{4}$, estos se caracterizan por su preocupación para hacer bien su trabajo en la atención con los presos con TB, llevando a cabo estrategias para el diagnóstico rápido y el correcto tratamiento de la enfermedad. Además, todos los profesionales sanitarios que ejecutaban las acciones para el control de la TB eran del área de enfermería, y estos profesionales, como se ha descrito, son los más involucrados en el control de la TB en otras penitenciarías ${ }^{18}$

Nuestro estudio cualitativo muestra que las acciones que llevan a cabo los profesionales en una prisión para acceder al diagnóstico del enfermo consisten en indagar en lo oculto y en las manifestaciones de la enfermedad que expresan los presos, con el fin de hallar signos y síntomas en el enfermo que accede a la enfermería. Estas acciones son similares a las que el MS caracteriza cómo acciones que se desarrollan para obtener el diagnóstico por demanda ambulatoria². Cuando los profesionales envían los papeles para saber los signos y síntomas que presentan los presos, estas son acciones que se caracterizan para el MS cómo acciones para el diagnóstico por búsqueda activa².

Es importante señalar, como lo indican los datos cualitativos, que no siempre los profesionales pueden llevar a cabo sus acciones para el diagnóstico, pues existen límites impuestos en sus acciones que les imposibilita realizarlas, tales como la restricción de acceso de los presos a la enfermería y los motivos de seguridad. Lo anterior limita el acceso de los profesionales a 
los presos, así como el acceso de los reclusos a la enfermería, pues ésta depende de que los agentes de seguridad les den esta oportunidad. Esta realidad también se reporta en otros países, como China, donde los servicios a la disposición de la población de penitenciarías también están sujetos verticalmente al control del Ministerio de Justicia, y requieren de una interfaz con el sector de salud ${ }^{19}$.

En un ambiente influenciado por relaciones de fuerza y de poder, los presos dependen de la empatía del agente de seguridad para que éste les ofrezca esa oportunidad de traslado a la enfermería ${ }^{7,9}$; estas condiciones llegan a retrasar el diagnóstico ${ }^{9}$. En nuestro estudio se mostró el trabajo que desarrollan los profesionales para sensibilizar a los agentes de seguridad y a los propios presos, de manera que se pueda facilitar el diagnóstico a través de la búsqueda activa de los casos y de los contactos.

Estas dificultades señaladas por los profesionales entrevistados pueden ser las mismas que enfrentan los profesionales de otras prisiones del Estado. Esto permite entender el por qué, según los resultados cuantitativos, "búsqueda activa" y "búsqueda activa de contactos", presentan un bajo porcentaje de casos diagnosticados, toda vez que, como es señalado por los profesionales sanitarios entrevistados, el desarrollo de las acciones para estas dos formas de obtención del diagnóstico son las que más dependen de una importante articulación con los profesionales de seguridad de las prisiones.

En un estudio desarrollado por una enfermera de un centro penitenciario de España, se resalta la importancia de que el recluso tenga acceso a profesionales sanitarios, principalmente por el hecho de tener un diagnóstico de enfermedades rápido. Lo anterior porque, en ciertas ocasiones, las prisiones se constituyen como la primera oportunidad de algunos individuos para recibir un servicio de cuidados en salud ${ }^{20}$.

Además, entender las dificultades de acceso a profesionales sanitarios nos puede ayudar a comprender por qué un porcentaje considerable de casos (5,5\%) que fueron diagnosticados en servicios de Urgencia/Emergencia, presentaron un agravamiento del cuadro de salud del preso enfermo que los condujo a necesitar de servicios más especializados para un diagnóstico más certero.

Es por eso que es importante el descubrimiento rápido de casos, principalmente hay predominio de los casos pulmonares, como lo muestran los datos cuantitativos. Como consecuencia, existe la necesidad de repensar la realización de estrategias de diagnóstico de TB en el sistema penitenciario, como, diagnóstico de la TB activa y/o latente en el momento de la entrada del preso en la prisión, aislar los presos en la enfermería en el momento de su entrada en la institución, hasta un período que posibilite al equipo de sanidad hacer las pruebas necesarias para el diagnóstico de la TB al preso enfermo, antes que empiece a compartir celda con los demás.

Aun así, diagnosticar de manera rápida a los enfermos de TB en prisiones, tanto como la identificación de la TB latente, constituye una importante estrategia de prevención del padecimiento de TB activa, toda vez que, con un enfoque preventivo y un diagnóstico temprano, el tratamiento latente permite evitar que los reclusos que poseen condiciones de salud o susceptibles al desarrollo de la enfermedad, la desarrollen de forma activa ${ }^{21}$.

A respecto de las condiciones de salud, es común que los presos presenten síntomas como la tos en el entorno penitenciario, debido a la drogadicción lícita e ilícita, por lo cual tiende a no valorarse este síntoma como un indicativo de la presencia de TB activa ${ }^{9}$. Además, se destaca el desconocimiento de los presos sobre los aspectos relacionados con la enfermedad y su tratamiento. Un estudio realizado en Etiopía reveló que la mayoría de los presos entrevistados desconocían lo siguiente: la causa de la enfermedad, y la relacionaban con el viento frío; la posibilidad de contraer bacilos multirresistentes en casos de no adherencia; y demás elementos relacionados a la enfermedad, lo que dificulta a la identificación y el tratamiento ${ }^{22}$.

Otros problemas de salud como la coinfección por VIH dificultan un diagnóstico certero de la TB, al presentar condiciones similares y síntomas característicos, como el adelgazamiento y la fiebre ${ }^{2}$, lo que trae consigo que los presos enfermos no les den importancia a estos síntomas. Es por esto que los profesionales de la prisión investigada realizan la estrategia de indagar en lo manifiesto, estando siempre atentos y solicitando a todos los reclusos los exámenes necesarios para el diagnóstico de la TB, incluso a aquellos que presentan sólo un signo y/o síntoma sugestivos de esa enfermedad.

Como se observa en los datos cuantitativos del período estudiado, se necesita más atención para los coinfectados con el VIH/sida, por presentar una tendencia decreciente entre los casos diagnosticados, lo que supone que las demás prisiones del Estado no valoran la oferta de pruebas para diagnosticar el $\mathrm{VIH} /$ sida en enfermos de TB. Además, diagnosticar el VIH/sida en presos con TB es importante, con el fin de tener seguimiento de la realización del tratamiento de estos enfermos, toda vez que las personas con $\mathrm{VIH} /$ sida se presentan como factores de riesgo para no completar el tratamiento de la TB en prisiones ${ }^{23}$.

Es importante destacar que la ocurrencia de $\mathrm{TB}$ y $\mathrm{VIH} /$ sida en las prisiones merece atención. En sistemas penitenciarios de diferentes países es difícil ofertar el tratamiento de una o ambas enfermedades. Se destaca la falta de provisión de medicamentos o regímenes terapéuticos específicos en prisiones, las barreras estructurales y las dificultades de los presos para revelar su estado de salud a los profesionales sanitarios de las prisiones ${ }^{24}$.

Aún sobre las condiciones de salud, el alcoholismo y uso de drogas ilícitas han presentado una tendencia creciente en el período estudiado, lo que supone a una mayor necesidad de atención al tratamiento del preso enfermo, pues se sabe que el uso de drogas es un factor que fragiliza la efectividad al tratamiento de la $\mathrm{TB}^{25}$. Esta es una condición que los profesionales sanitarios de la prisión estudiada destacan sobre los esfuerzos que hacen para mantener el tratamiento del preso enfermo, ya para ello ofrecen orientaciones sobre adquirir hábitos saludables y disminuir el uso de drogas, para lograr la curación.

EI TDO y orientaciones sobre el tratamiento hasta su finalización, son las principales estrategias recomendadas por la Organización Mundial de la Salud (OMS) para el desarrollo 
del tratamiento de enfermos por TB en prisiones, con la finalidad de realizar un seguimiento adecuado y evitar el abandono del tratamiento ${ }^{2}$. Una vez realizado de la manera recomendada por el MS y la OMS ${ }^{1,2}$, el TDO es una herramienta importante para identificar casos de personas que aún están enfermas, lo que hace relevante una mayor atención a las condiciones de salud de éstos.

Según los resultados cualitativos, el inicio del tratamiento de la TB en la prisión investigada se realiza cuando el enfermo está aislado en la enfermería; no obstante, el mantenimiento de manera ambulatoria sin el TDO difiere del recomendado por el MS y la OMS, quienes recomiendan ofrecerlo durante todo el período del esquema terapéutico. Este hallazgo conlleva a cuestionar la fiabilidad, lo que se señala en los datos cuantitativos: si del $90 \%$ de los casos, se realizó efectivamente el TDO.

Aún más, en la prisión estudiada, por tener conocimiento de que el tratamiento con TDO sólo se realiza durante el aislamiento del enfermo en la enfermería, los profesionales utilizan otras estrategias como la coerción, usada en diferentes prisiones alrededor del mundo para intentar garantizar el cumplimiento del tratamiento?.

Se reconoce que otras prisiones de Brasil y otros países, también enfrentan dificultades para realizar el TDO9,26, por dificultades en disponer de los recursos humanos necesarios ${ }^{9}$, y la dificultad de articular el equipo de seguridad y el acceso del preso enfermo a un equipo sanitario. Por esa dificultad se debería valorar la necesidad de priorizar eITDO en prisiones al menos a los casos que poseen mayores probabilidades de abandono ${ }^{20}$ como aquellos de VIH y casos de drogadicción lícita o ilícita.

Para la finalización del tratamiento, en el panorama cuantitativo investigado, la curación se mantuvo superior a los $85 \%$ de los casos y presentó una tendencia creciente, siendo el abandono y el óbito no-TB decrecientes. Los profesionales sanitarios se involucran en hacer bien el trabajo que desempeñan, pero demuestran una preocupación por controlar esta enfermedad. Se debe resaltar que la SAP da una gratificación a las prisiones que alcanzan metas como es la de mayor porcentaje de curación de la TB. ${ }^{4}$ Esas iniciativas de motivación han influenciado en el aumento de la curación de la TB y la disminución del abandono del tratamiento.

Con el desarrollo de esta investigación, se puede notar que para acceder al control de la TB en una prisión, es de gran importancia el empeño de los profesionales sanitarios, principalmente del área de enfermería. Sin el compromiso por parte de los profesionales para el control de dicha enfermedad, el retraso de diagnósticos y la dificultad para cumplir con el tratamiento, son barreras que pueden seguir ocurriendo y perpetuándose como parte de las dificultades para el control de la TB en un sistema penitenciario.

El estudio presenta limitaciones debido a que los datos cuantitativos de la fase I fueron extraídos de fuente secundaria y que, por lo tanto pueden contener errores o supresión. Además, la fase II se realizó en una única prisión; es posible que otras prisiones de Brasil u otros países obtengan resultados diferentes por presentar condiciones distintas.
Aún más, por motivos de las obligaciones con los requisitos que se piden en los trámites de los Comités Éticos de la Institución de Educación Superior, y de la SAP, la prisión estudiada en la fase Il fue elegida previamente a la obtención de los datos de la fase I, lo que significa que los análisis de la fase I fueron realizados ya con la prisión de la fase II escogida, lo que limitó realizar análisis más profundos en la fase I para dirigir la realización de la fase II para otras prisiones.

\section{CONCLUSIONES E IMPLICACIONES PARA LA PRÁCTICA}

Esta investigación de métodos mixtos posibilitó la comprensión de cómo está el desarrollo del control de la TB en un sistema penitenciario brasileño. La situación de la TB en el sistema penitenciario estudiado es importante. La mayoría fueron casos pulmonares y obtuvieron diagnóstico a través de la demanda ambulatoria. A pesar de que la curación presenta una tendencia creciente en el período del 2010 al 2016, la presencia del VIH/ sida y el uso de drogas lícitas e ilícitas se convierten en desafíos para el diagnóstico y tratamiento. Así que, el empeño de los profesionales sanitarios para mantener el desarrollo constante de las estrategias que posibiliten el diagnóstico y tratamiento, auxilia para un control de la TB en estas Instituciones.

Sin embargo, hace falta destacar que, ante las dificultades que tienen los profesionales sanitarios para desarrollar las acciones para el diagnóstico y tratamiento identificados en el estudio, se necesitan más investigaciones que permitan comprender y poner en marcha estrategias para el control de la TB que resulten más eficaces en los sistemas penitenciarios. Como parte de dichas investigaciones, se destaca el personal de enfermería, ya que son ellos los más involucrados en el control de la enfermedad en estos escenarios.

\section{FINANCIAMENTO}

Coordenação de Aperfeiçoamento de Pessoal de Nível Superior (CAPES) - Brasil. Código de Financiamento 001, bolsa de Doutorado, concedido a Mônica Cristina Ribeiro Alexandre d'Auria de Lima, primeira autora, no período de setembro de 2015 a setembro de 2018.

\section{CONTRIBUICCIONES DE LOS AUTORES}

Desenho do estudo. Mônica Cristina Ribeiro Alexandre d'Auria de Lima.

Coleta ou produção dos dados. Mônica Cristina Ribeiro Alexandre d'Auria de Lima.

Análise de dados. Mônica Cristina Ribeiro Alexandre d'Auria de Lima. Maria Mercedes Martinez-Marcos. Jaqueline Garcia de Almeida Ballestero. Teresinha Heck Weiller. Cassiara Boeno Borges de Oliveira. Pedro Fredemir Palha.

Interpretação dos resultados. Mônica Cristina Ribeiro Alexandre d'Auria de Lima. Maria Mercedes Martinez-Marcos. Jaqueline 
Garcia de Almeida Ballestero. Teresinha Heck Weiller. Cassiara Boeno Borges de Oliveira. Pedro Fredemir Palha.

Redação e revisão crítica do manuscrito. Mônica Cristina Ribeiro Alexandre d'Auria de Lima. Maria Mercedes Martinez-Marcos. Jaqueline Garcia de Almeida Ballestero. Teresinha Heck Weiller. Cassaria Boeno Borges de Oliveira. Pedro Fredemir Palha.

Aprovação da versão final do artigo. Mônica Cristina Ribeiro Alexandre d'Auria de Lima. Maria Mercedes Martinez-Marcos. Jaqueline Garcia de Almeida Ballestero. Teresinha Heck Weiller. Cassiara Boeno Borges de Oliveira. Pedro Fredemir Palha.

Responsabilidade por todos os aspectos do conteúdo e a integridade do artigo publicado. Mônica Cristina Ribeiro Alexandre d'Auria de Lima. Maria Mercedes Martinez-Marcos. Jaqueline Garcia de Almeida Ballestero. Teresinha Heck Weiller. Cassiara Boeno Borges de Oliveira. Pedro Fredemir Palha.

\section{EDITOR ASSOCIADO}

\author{
Cristina Rosa Baixinho (D)
}

\section{EDITOR CIENTÍFICO}

\author{
Ivone Evangelista Cabral (D)
}

\section{REFERENCIAS}

1. World Health Organization. Global tuberculosis report 2019 [Internet] Geneva:WHO; 2019 [citado 2019 jul 10]. Disponible en: https://www. who.int/tb/publications/global_report/en/

2. Ministério da Saúde (BR). Manual de recomendações para o controle da tuberculose no Brasil [Internet]. 2. ed. Brasília: MS; 2019 [citado 2019 abr 10]. Disponible en: https://sbpt.org.br/portal/manual-controletuberculose/

3. Souza KMJ, Villa TCS, Assolini FEP, Beraldo AA, França UM, Protti $\mathrm{ST}$ et al. Atraso no diagnóstico da tuberculose em sistema Prisional: a experiência do doente apenado. Texto Contexto Enferm. 2012;21(1):1725. http://dx.doi.org/10.1590/S0104-07072012000100002.

4. Ministério da Justiça (BR), Departamento Penitenciário Nacional. Levantamento nacional de informações penitenciárias INFOPEN dezembro de 2016 [Internet]. Brasília: MJ; 2017 [citado 2019 abr 10]. Disponible en: http://www.justica.gov.br/noticias/mj-divulgara-novorelatorio-do-infopen-nesta-terca-feira/relatorio-depen-versao-web.pdf

5. Bourdillon PM, Gonçalves CCM, Pelissari DM, Arakaki-Sanchez D, Ko Al, Croda J et al. Increase in tuberculosis cases among prisoners, Brazil, 2009-2014. Emerg Infect Dis. 2017;23(3):496-9. http://dx.doi. org/10.3201/eid2303.161006. PMid:28221118.

6. Pelissari DM, Kuhleis DC, Bartholomay P, Barreira D, Oliveira CLP, de Jesus RS et al. Prevalence and screening of active tuberculosis in a prison in the South of Brazil. Int J Tuberc Lung Dis. 2018;22(10):116671. http://dx.doi.org/10.5588/ijtld.17.0526. PMid:30236184.

7. Ferreira PG, Ferreira AJ, Cravo-Roxo P. Constrangimentos ao controlo da tuberculose no sistema prisional. Rev. Port. Sau. Pub. 2015;33(1):7183. http://dx.doi.org/10.1016/j.rpsp.2014.11.003.

8. Valença MS, Possuelo LG, Cezar-Vaz MR, Silva PEA. Tuberculose em presídios brasileiros: uma revisão integrativa da literatura. Cien Saude Colet. 2016;21(7):2147-60. http://dx.doi.org/10.1590/141381232015217.16172015. PMid:27383348.
9. Valença MS, Cezar-Vaz MR, Brum CB, Silva PEA. O processo de detecção e tratamento de casos de tuberculose em um presídio. Cien Saude Colet. 2016;21(7):2111-22. http://dx.doi.org/10.1590/141381232015217.13822015. PMid:27383345.

10. Zulay PP. Los diseños de método mixto en la investigación en educación: una experiencia concreta. Rev Electróni Educar [Internet]. 2011; [citado 2019 ene 20];15(1):15-29. Disponible en: https://www.redalyc.org/ articulo.oa? id=194118804003

11. Creswell JW, Clark VLP. Pesquisa de métodos mistos. 2. ed. Porto Alegre: Penso; 2013.

12. Antunes JL, Cardoso MR. Uso da análise de séries temporais em estudos epidemiológicos. Epidemiol Serv Saude. 2015;24(3):565-76. http://dx.doi.org/10.5123/S1679-49742015000300024.

13. Martínez-Salgado C. El muestreo en investigación cualitativa: principios básicos y alguna controversia. Cien Saude Colet. 2012;17(3):613-9. http://dx.doi.org/10.1590/S1413-81232012000300006. PMid:22450401.

14. Charmaz K. Constructing grounded theory. Thoausand Oaks: SAGE 2014.

15. Morse JM. Using shadowed data. Qual Health Res. 2001;11(3):291-2 http://dx.doi.org/10.1177/104973201129119091. PMid:11339074.

16. Martinez-Marcos M. Un estudio cualitativo sobre mujeres cuidadoras con enfermedad crónica [tese]. Alicante: Universidad de Alicante; 2015 [citado 2019 abr 10]. Disponible en: http://rua.ua.es/dspace/ handle/10045/54216

17. Wallis RS, Maeurer M, Mwaba P, Chakaya J, Rustomjee R, Migliori GB et al. Tuberculosis: advances in developmentof new drugs, treatmentregimens, host-directedtherapies, and biomarkers. Lancet Infect Dis. 2016;16(4):e34-46. http://dx.doi.org/10.1016/S14733099(16)00070-0. PMid:27036358.

18. Sicsú AN, Gonzales RIC, Mitano F, Sousa LO, Silva LMCD, Ballestero JGA et al. Nursing practices centered on individuals with tuberculosis: an interface with democracy. Rev Bras Enferm. 2019;72(5):1219-25. http://dx.doi.org/10.1590/0034-7167-2017-0380. PMid:31531644.

19. Tong Y, Jiang S, Guan X, Hou S, Cai K, Tong Y et al. Epidemic situation of tuberculosis in prisons in the central region of China. Am J Trop Med Hyg. 2019;101(3):510-2. http://dx.doi.org/10.4269/ajtmh.18-0987. PMid:31287047.

20. Chaer-Yemlahi SS. Enfermería penitenciaria, la gran desconocida. Metas Enferm [Internet]. 2018; [citado 2021 jun 12];21(7):62-6. Disponible en: https://dialnet.unirioja.es/servlet/articulo?codigo $=6534823$

21. Godoy P. Directrices sobre el control de la infección tuberculosa latente para apoyar la eliminación de la tuberculosis. Rev Esp Sanid Penit. 2021;23(1):29-38. http://dx.doi.org/10.18176/resp.00028. PMid:33847703.

22. Adane K, Spigt M, Johanna L, Noortje D, Abera SF, Dinant GJ. Tuberculosis knowledge, attitudes, and practices among northern Ethiopian prisoners: implications for TB control efforts. PLoS One. 2017;12(3):e0174692. http://dx.doi.org/10.1371/journal.pone.0174692. PMid:28358877.

23. Chong F, Marín D, Pérez F. Low detection rate and therapeutic success of tuberculosis in a prison in Ecuador. Rev Panam Salud Publica. 2019;43:e106. http://dx.doi.org/10.26633/RPSP.2019.106. PMid:31908648.

24. Herce ME, Muyoyeta M, Topp SM, Henostroza G, Reid SE. Coordinating the prevention, treatment, and care continuum for HIV-associated tuberculosis in prisons: a health systems strengthening approach. Curr Opin HIV AIDS. 2018;13(6):492-500. http://dx.doi.org/10.1097/ $\mathrm{COH} .0000000000000505$. PMid:30222608.

25. Santos MNA, Sá AMM. O ser-portador de tuberculose em prisões: um estudo de enfermagem. Esc Anna Nery. 2014;18(2):350-5. http:// dx.doi.org/10.5935/1414-8145.20140051.

26. Gupta RK, Lipman M, Story A, Hayward A, de Vries G, van Hest R et al. Active case finding and treatment adherence in risk groups in the tuberculosis pre-elimination era. Int J Tuberc Lung Dis. 2018;22(5):47987. http://dx.doi.org/10.5588/ijtld.17.0767. PMid:29663951. 